\title{
Surface gap solitons at a nonlinearity interface
}

\author{
Tomáš Dohnal ${ }^{1}$ and Dmitry Pelinovsky ${ }^{2 *}$ \\ ${ }^{1}$ Seminar for Applied Mathematics, ETH Zürich, Switzerland \\ ${ }^{2}$ Institut für Analysis, Dynamik und Modellierung, Universität Stuttgart, Germany
}

November 21, 2018

\begin{abstract}
We demonstrate existence of waves localized at the interface of two nonlinear periodic media with different coefficients of the cubic nonlinearity via the one-dimensional Gross-Pitaevsky equation. We call these waves the surface gap solitons (SGS). In the case of smooth symmetric periodic potentials, we study analytically bifurcations of SGS's from standard gap solitons and determine numerically the maximal jump of the nonlinearity coefficient allowing for the SGS existence. We show that the maximal jump vanishes near the thresholds of bifurcations of gap solitons. In the case of continuous potentials with a jump in the first derivative at the interface, we develop a homotopy method of continuation of SGS families from the solution obtained via gluing of parts of the standard gap solitons and study existence of SGS's in the photonic band gaps. We explain the termination of the SGS families in the interior points of the band gaps from the bifurcation of linear bound states in the continuous non-smooth potentials.
\end{abstract}

\section{Introduction}

We are concerned with localized waves at the interface of two periodic nonlinear media called surface gap solitons (SGS). One of the first publications on optical solitons propagating along material interfaces is [17], where the interface of a linear and a focusing Kerr nonlinear medium is studied. In the last two years relevant publications in the context of nonlinear optics have dealt, for instance, with discrete surface solitons in nonlinear waveguide

${ }^{*}$ On leave from Department of Mathematics, McMaster University, Hamilton, Ontario, Canada, L8S 4K1 
arrays [4, 10, 16, surface gap solitons at the interface of a uniform and a periodic medium with the defocusing cubic nonlinearity [7] and surface vortex solitons at the interface of two periodic media with different mean values of the refractive index and with the saturable nonlinearity [5, 6]. One of the typical models employed in the theory of gap solitons is the one-dimensional nonlinear Schrödinger equation (NLS) with cubic nonlinearity and periodic potential called the Gross-Pitaevsky equation.

We investigate here the existence of surface waves at the interface of two media with identical periodic linear parts of the refractive index and with different cubic nonlinearities. It is known that for most photonic materials a variation in the nonlinear part of the refractive index $n_{2}$ is necessarily accompanied by a larger change in the linear part $n_{0}$. Nevertheless, certain materials exhibit large variations in $n_{2}$ accompanied by small variations in $n_{0}$, see [1, 15. Localized states have been studied theoretically in media with constant $n_{0}$ and spatially periodic $n_{2}$ in [3].

Each of the two periodic nonlinear media supports at least two families of standard gap solitons in every bounded non-empty frequency gap. One family is always unstable, while the other can be stable depending on the locations of spectral bands and bifurcations of eigenvalues from the band edges [13]. The potentially stable family looks like a single-humped envelope soliton with exponential decay and oscillations near the central peak. Multihumped envelope solitons may also exist in such periodic nonlinear media but we shall focus herein on existence of a single-humped solution localized near the interface between the two periodic nonlinear media.

The paper is organized as follows. Section 2 reviews Floquet theory for the governing Gross-Pitaevsky equation and summarizes the results on existence of gap solitons. In Section 3 we study the existence of SGS's for a smooth symmetric periodic potential function and find the maximal allowed jump in the nonlinearity coefficient between the two media for existence of SGS's. Section 4 discusses bifurcations and existence of SGS's for a continuous potential function with a derivative jump at the nonlinearity interface. Section 5 concludes the paper with conjectures on stability of SGS's. 


\section{Background: Floquet theory and gap soliton ex- istence}

We consider the one-dimensional periodic cubic Schrödinger equation in the form

$$
i u_{t}=-u_{x x}+V(x) u-\Gamma(x)|u|^{2} u, \quad x \in \mathbb{R}, \quad t \geq 0,
$$

where $x$ and $t$ are the spatial and temporal variable respectively, $V(x)$ is a real, continuous and $d$-periodic potential, and $\Gamma(x)=\Gamma_{ \pm}$for $\pm x>0$ is a real nonlinearity coefficient with constants $\Gamma_{+}$and $\Gamma_{-}$. The positive values of $\Gamma(x)$ corresponds to the focusing nonlinearity and the negative values of $\Gamma(x)$ to defocusing nonlinearity.

We are interested in the existence of stationary solutions of (2.1) localized near the interface at $x=0$ and having the form

$$
u(x, t)=e^{-i \omega t} \phi(x) \quad \text { s.t. } \quad \phi: \mathbb{R} \rightarrow \mathbb{R}, \quad \phi \rightarrow 0 \text { as }|x| \rightarrow \infty .
$$

The function $\phi(x)$ has to satisfy the second-order non-autonomous ODE

$$
-\phi^{\prime \prime}-\omega \phi+V(x) \phi-\Gamma(x) \phi^{3}=0,
$$

which can be cast in the Hamiltonian form with the Hamiltonian function

$$
H[\phi]=\frac{1}{2}\left[\left(\phi^{\prime}\right)^{2}+\omega \phi^{2}-V(x) \phi^{2}\right]+\frac{1}{4} \Gamma(x) \phi^{4} .
$$

Since $\Gamma(x)$ is discontinuous at $x=0, \phi(x)$ is a weak solution of the ODE (2.3) in $\phi \in C^{2}\left(\mathbb{R}_{+} \cup \mathbb{R}_{-}\right)$, such that the second derivative $\phi^{\prime \prime}(x)$ may have a jump at $x=0$. The continuously differentiable solution $\phi \in C^{1}(\mathbb{R})$ is a critical point of the energy functional

$$
E_{\omega}[\phi]=\frac{1}{2} \int_{\mathbb{R}}\left[-\left|\phi^{\prime}\right|^{2}+\omega|\phi|^{2}-V(x)|\phi|^{2}\right] d x+\frac{1}{4} \int_{\mathbb{R}} \Gamma(x)|\phi|^{4} d x,
$$

such that the first variation of $E_{\omega}^{\prime}[\phi]$ recovers the ODE (2.3).

Replacing $t$ by $z$ in (2.1) and (2.2), the $x$-localized solution $u(x, z)$ can be viewed as a spatial soliton propagating along the direction $z$ and localized in the transverse direction $x$ (localization in the third spatial direction $y$ is assumed to be achieved via total internal reflection). The parameter $\omega$ plays a role of the propagation constant. As we show below, the localized solutions of the ODE (2.3) decay exponentially as $|x| \rightarrow \infty$ only if $\omega$ belongs to the frequency gaps in the continuous spectra of the operator $L:=-\partial_{x x}+V(x)$ 
called the photonic band gaps. To do so, we recall the basic Floquet theory (see [2, 9]) for the Hill's equation

$$
L \psi(x)=-\psi^{\prime \prime}(x)+V(x) \psi(x)=\omega \psi(x), \quad x \in \mathbb{R} .
$$

The bounded solutions $\psi(x)$ of the Hill's equation (2.5) are usually called Bloch functions. Given a real, continuous and $d$-periodic potential $V(x)$, bounded solutions $\psi(x)$ exist for $\omega$ in a union of (possibly disjoint) spectral bands from

$$
\Sigma:=\left[\omega_{0}, \omega_{1}\right] \cup\left[\omega_{2}, \omega_{3}\right] \cup\left[\omega_{4}, \omega_{5}\right] \cup \ldots,
$$

where $\omega_{2 n-2}<\omega_{2 n-1} \leq \omega_{2 n}, n \in \mathbb{N}$ and $\omega_{n} \rightarrow \infty$ as $n \rightarrow \infty$. The set $\Sigma$ represents the complete (purely continuous) spectrum of the operator $L$ [2]. We shall assume for simplicity that all spectral bands are disjoint with $\omega_{2 n-1}<\omega_{2 n}, n \in \mathbb{N}$, such that all finite frequency gaps are non-empty.

For a fixed $\omega$ in the interior point of $\Sigma$, both fundamental solutions of the second-order ODE (2.5) are quasi-periodic in $x$ and have the representation $\psi=p_{ \pm}(x) e^{ \pm i k x}$, where $p_{ \pm}(x)=p_{ \pm}(x+d)$ and $k \in\left[0, \frac{\pi}{d}\right]$. The parameter $k$ parameterizes the frequency parameter $\omega$, such that we shall use notation $\omega=\omega_{2 n, 2 n+1}(k)$ for the spectral band in $\omega \in\left[\omega_{2 n}, \omega_{2 n+1}\right]$. If the $n$-th band is separated from the $(n+1)$-th band (i.e. $\omega_{2 n-1}<\omega_{2 n}$ and $\omega_{2 n+1}<\omega_{2 n+2}$ ), then $\omega_{2 n, 2 n+1}^{\prime}(k)=0$ and $\omega_{2 n, 2 n+1}^{\prime \prime}(k) \neq 0$ at the end points $k=0$ and $k=\frac{\pi}{d}$ [8].

When $\omega=\omega_{n}$, one of the solutions $\psi=\psi_{n}(x)$ is either $d$-periodic (corresponding to $k=0$ ) or $d$-antiperiodic (corresponding to $k=\frac{\pi}{d}$ ) and the other fundamental solution $\psi(x)$ grows linearly in $x$. For a fixed $\omega \in \mathbb{R} \backslash \Sigma$ the two fundamental solutions of (2.5) grow exponentially either in $x$ or $-x$ and have the representation $\psi=u_{ \pm}(x) e^{ \pm \kappa x}$, where $u_{ \pm}(x)$ is either periodic or anti-periodic and $\kappa=\kappa(\omega) \in \mathbb{R}_{+}$. The functions $u_{ \pm}(x)$ are periodic (anti-periodic if the bounded solutions $\psi_{n}(x)$ are periodic (anti-periodic) at the band edges $\omega_{2 n-1}$ and $\omega_{2 n}$, which surround the band gap.

Suppose that $\phi(x)$ is a localized solution of the ODE (2.3). It is then obvious from the linearized analysis that the solution $\phi(x)$ decays exponentially as $|x| \rightarrow \infty$ only if $\omega \in \mathbb{R} \backslash \Sigma$. It was shown under fairly general assumptions (see [13] and references therein) that the families of gap solitons of the ODE (2.3) with constant coefficient $\Gamma(x)=\Gamma_{0}$ undertake a local bifurcation from all points $\omega=\omega_{2 m}, m \geq 0$ to the left if $\Gamma_{0}>0$ and from all points $\omega=\omega_{2 m+1}, m \geq 0$ to the right if $\Gamma_{0}<0$ (the term local bifurcation means that $\|\phi\|_{L^{\infty}} \rightarrow 0$ as $\left.\omega \rightarrow \omega_{n}\right)$. This conjecture was rigorously proved in [11], where existence of exponentially decaying gap solitons in $H^{1}(\mathbb{R})$ was confirmed in every finite frequency gap $\omega \in\left(\omega_{2 m-1}, \omega_{2 m}\right), m \in \mathbb{N}$ and in 
the semi-infinite frequency gap $\omega<\omega_{0}$ for $\Gamma_{0}>0$. We use this result but simplify our consideration by working with the class of symmetric potentials $V=V_{0}(x)$, where $V_{0}(-x)=V_{0}(x)$ on $x \in \mathbb{R}$. In particular, we shall perform numerical computations with

$$
V_{0}(x)=\sin ^{2}\left(\frac{\pi x}{d}\right), \quad d=10,
$$

which has a minimum at $x=0$, i.e. at our interface location. The spectral bands and gaps of $V_{0}(x)$ are approximated numerically from the Hill's equation (2.5). For instance, the first five band edges of the potential (2.6) are located as follows

$$
\omega_{0} \approx 0.283, \quad \omega_{1} \approx 0.291, \quad \omega_{2} \approx 0.747, \quad \omega_{3} \approx 0.843, \quad \omega_{4} \approx 1.057 .
$$

As partly seen in Fig. 1 of [13], for $V_{0}(x)=\sin ^{2}(\pi x / 10)$ the Bloch functions $\psi=\psi_{n}(x)$ at the first eight band edges $\omega=\omega_{n}, n \in\{0,1, \ldots, 7\}$ have the following symmetry properties:

$$
\begin{aligned}
& \psi_{n}(-x)=\psi_{n}(x), n \in\{0,1,4,5\}, \\
& \psi_{n}(-x)=-\psi_{n}(x), n \in\{2,3,6,7\} .
\end{aligned}
$$

Clearly, all $\psi_{n}(x)$ must be even or odd since the Hill's equation (2.5) is symmetric with respect to reflection $x \mapsto-x$ and admits only one linearly independent bounded eigenfunction $\psi=\psi_{n}(x)$ at $\omega=\omega_{n}$. Nevertheless, for other even potentials $V_{0}(x)$ the ordering between even and odd Bloch functions can be different than in (2.7) and the only statement about this ordering that is valid for general even $V_{0}(x)$ can be deduced from Theorem 3.1.1 in [2], which says that two subsequent even eigenfunctions have to be followed by an odd one and vice versa. We refrain from such a discussion here concentrating on the potential (2.6), for which symmetries (2.7) hold.

In contrast to the ordering between even and odd Bloch functions, the ordering between $d$-periodic and $d$-antiperiodic Bloch functions is unique for all $d$-periodic $V_{0}(x)$ (not necessarily even). By the trace of the monodromy matrix [2], the periodic functions $\psi_{n}(x)$ correspond to the set $n \in S_{+}$ with $S_{+}=\{0,3,4,7,8, \ldots\}=\{0,4 k-1,4 k ; k \in \mathbb{N}\}$ and the anti-periodic functions $\psi_{n}(x)$ correspond to the set $n \in S_{-}$with $S_{-}=\{1,2,5,6, \ldots\}=$ $\{4 k-3,4 k-2 ; k \in \mathbb{N}\}$.

By Sturm's Theorem (see Theorem 3.1.2 in [2]) for general $d$-periodic $V_{0}(x)$ the periodic functions $\psi_{n}(x)$ with $n \in S_{+}$have no nodes on $x \in[0, d)$ for $n=0$, two nodes for $n=3,4$, four nodes for $n=7,8$, etc. Similarly, the 


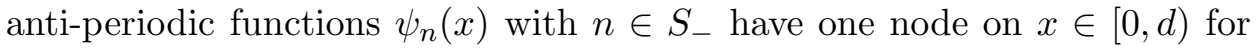
$n=1,2$, three nodes for $n=5,6$, etc.

Altogether, this set of facts about Bloch functions at the first eight band edges for the potential (2.6) is summarized in Table 1.

\begin{tabular}{||c||c|c|c|c|c|c|c|c||}
\hline$n$ & 0 & 1 & 2 & 3 & 4 & 5 & 6 & 7 \\
\hline symmetry & even & even & odd & odd & even & even & odd & odd \\
\hline periodicity & $S_{+}$ & $S_{-}$ & $S_{-}$ & $S_{+}$ & $S_{+}$ & $S_{-}$ & $S_{-}$ & $S_{+}$ \\
\hline$\#$ nodes on $[0, d)$ & 0 & 1 & 1 & 2 & 2 & 3 & 3 & 4 \\
\hline sign of $\Gamma_{0}$ for local bifurcation & 1 & -1 & 1 & -1 & 1 & -1 & 1 & -1 \\
\hline
\end{tabular}

Table 1: Properties of the Bloch functions $\psi_{n}(x)$ and gap soliton bifurcations at the first eight band edges of the even potential $V_{0}(x)=\sin ^{2}(\pi x / 10)$.

Let $\phi_{0}(x)$ be a single-humped solution of the ODE (2.3) with $\Gamma(x)=\Gamma_{0}$ and $V(x)=V_{0}(x)=\sin ^{2}(\pi x / 10)$ which bifurcates from the band edge $\omega=$ $\omega_{n}$. By the local bifurcation theory [13, it inherits the symmetry properties (2.7) of the Bloch function $\psi_{n}(x)$. Therefore, $\phi_{0}(-x)=\phi_{0}(x)$ for branches of gap solitons to the left of $\omega_{n}$ with $n=\{0,4\}$ (for $\Gamma_{0}>0$ ) and to the right of $\omega_{n}$ with $n=\{1,5\}$ (for $\Gamma_{0}<0$ ), while $\phi_{0}(-x)=-\phi_{0}(x)$ for branches of gap solitons to the left of $\omega_{n}$ with $n=\{2,6\}$ (for $\Gamma_{0}>0$ ) and to the right of $\omega_{n}$ with $n=\{3,7\}$ (for $\Gamma_{0}<0$ ). See Figs. 2-3 in [13] for gap solitons $\phi_{0}(x)$ in the potential (2.6).

In this paper, we shall consider existence of surface gap solitons in the ODE (2.3) with piecewise constant coefficient $\Gamma(x)=\Gamma_{ \pm}$for $\pm x>0$ and potential $V(x)$ of the following two classes:

$$
\text { (i) } V=V_{0}(x), \quad \text { (ii) } V=V_{0}(x-\delta) \chi_{(-\infty, 0)}+V_{0}(x+\delta) \chi_{[0, \infty)} \text {, }
$$

where $\chi_{[a, b]}=1$ on $x \in[a, b]$ and zero otherwise, while $0<\delta<d$. Here $V_{0}(x)$ is a smooth, even, $d$-periodic function on $x \in \mathbb{R}$. We note that $V(x)$ in (ii) is continuous and even on $x \in \mathbb{R}$ but smooth and periodic only on each $\pm x>0$.

One can develop a general shooting method for numerical approximations of SGS's from the condition that a localized solution $\phi(x)$ of the second-order ODE (2.3) with $\omega \in\left(\omega_{2 m-1}, \omega_{2 m}\right), m \in \mathbb{Z}$ decays to zero at infinity according to two fundamental solutions $p_{ \pm}(x) e^{\mp \kappa x}$ as $x \rightarrow \pm \infty$, where $\kappa=\kappa(\omega)$ is a positive number. Solving the ODE (2.3) with $\Gamma(x)=\Gamma_{+}$ for a general initial value $\phi(0)$ and $\phi^{\prime}(0)$ to $x>0$ and the same ODE with 
$\Gamma(x)=\Gamma_{-}$to $x<0$, one can construct a continuously differentiable solution $\phi(x)$ on $x \in \mathbb{R}$ which decays to zero as $x \rightarrow \pm \infty$ if and only if the projections to the growing fundamental solutions $p_{ \pm}(x) e^{ \pm \kappa x}$ are zero at infinity. The system of two constraints for two initial values constitute a well-posed problem of numerical analysis. However, practical implementations of this algorithm are unclear as the shooting method may depend sensitively on starting approximations of the initial value and may require long computations time to search through all appropriate initial values. In addition, the ODE solvers of the shooting method may develop numerical instabilities in approximations of growing solutions.

Due to these reasons, we shall develop an alternative view on numerical approximations of SGS's, starting with local bifurcation analysis and using the homotopy continuation method to trace the solution families along parameters $\omega, \Gamma_{ \pm}$and $\delta$. Using these analytical and numerical results, we have obtained the following main results of the article.

1. We prove analytically that any gap soliton for $\Gamma_{+}=\Gamma_{-}$can be continued to the SGS for sufficiently small $\left|\Gamma_{+}-\Gamma_{-}\right|$under a non-degeneracy assumption.

2. We prove analytically that the maximal difference $\left|\Gamma_{+}-\Gamma_{-}\right|$leading to SGS existence converges to 0 when $\omega$ approaches the band edge which features the local bifurcation of a gap soliton.

3. Surface gap solitons are computed numerically when the potential $V(x)$ is given by (2.8) (i), and the maximal $\left|\Gamma_{+}-\Gamma_{-}\right|$allowing their existence is found. Our numerical results confirm the analytical results (1-2) above.

4. Existence of SGS's for $V(x)$ in (2.8) (ii) with $\Gamma_{+}>0$ and $\Gamma_{-}<0$ is studied. We show numerically that local bifurcations may occur from a countable set of points in the parameter domain $(\omega, \delta) \in$ $\left(\omega_{2 m-1}, \omega_{2 m}\right) \times(0, d), m \in \mathbb{N}$.

5. We compute numerically the points of local bifurcation of SGS's for the potential (2.8) (ii) and use the homotopy continuation of the bifurcating solution. As a result, we show that the family of SGS's exists typically in a subset of the plane $(\omega, \delta)$.

6. We show analytically that the termination of families of SGS's for the potential (2.8) (ii) is related to existence of linear bound states for the non-smooth potential. 
Results (1-3) are reported in Section 3 and results (4-6) are described in Section 4 .

\section{Bifurcations of surface gap solitons for smooth potentials}

In this section we study continuation of SGS's from gap solitons existing for $\Gamma_{+}=\Gamma_{-}$in the case of a smooth potential function $V(x)$. A prototypical example of such potential is the symmetric function $V_{0}(x)$ in (2.8) (i).

\subsection{Existence of Bifurcations from Gap Solitons}

Let $\gamma=\left(\Gamma_{+}+\Gamma_{-}\right) / 2$ and $\nu=\left(\Gamma_{+}-\Gamma_{-}\right) / 2$. Then, the ODE (2.3) can be rewritten in the form

$$
F(\phi, \nu)=-\phi^{\prime \prime}-\omega \phi+V(x) \phi-\gamma \phi^{3}-\nu \operatorname{sign}(x) \phi^{3}=0,
$$

where $F(\phi, \nu): H^{1}(\mathbb{R}) \times \mathbb{R} \mapsto H^{-1}(\mathbb{R})$ is a nonlinear operator acting on a function $\phi(x)$ in space $\phi \in H^{1}(\mathbb{R})$ and parameter $\nu \in \mathbb{R}$.

We assume that there exists a solution $\phi_{0}(x) \in H^{1}(\mathbb{R})$ for $\omega \in \mathbb{R} \backslash \Sigma$ and some $\gamma$ and $V(x)$, such that $F\left(\phi_{0}, 0\right)=0$. The Jacobian $D_{\phi} F\left(\phi_{0}, 0\right)$ is given by the Schrödinger operator $\mathcal{L}: H^{2}(\mathbb{R}) \mapsto L^{2}(\mathbb{R})$, where

$$
\mathcal{L}=-\partial_{x}^{2}-\omega+V(x)-3 \gamma \phi_{0}^{2}(x) .
$$

Since $\omega \in \mathbb{R} \backslash \Sigma$, we have $\phi_{0}^{2}(x) \rightarrow 0$ exponentially fast as $|x| \rightarrow \infty$, such that the term $-3 \gamma \phi_{0}^{2}(x)$ is a relatively compact perturbation to the unbounded operator $L-\omega$, where $L=-\partial_{x}^{2}+V(x)$. By a standard argument (see Corollary 2 in Section XIII.4 in [14]), the essential spectrum of $\mathcal{L}$ and $(L-\omega)$ coincide. Since $\omega \in \mathbb{R} \backslash \Sigma$, the zero point is isolated from the essential spectrum of $\mathcal{L}$. If we further assume that $\mathcal{L}$ has the trivial kernel in $H^{1}(\mathbb{R})$, then $\mathcal{L}$ is invertible on $L^{2}(\mathbb{R})$. Since the translational invariance is broken if $V(x) \neq 0, \mathcal{L}$ generally has the trivial kernel, unless a bifurcation of branches of gap solitons occur. By the standard analysis based on the Implicit Function Theorem, there exists a unique smooth continuation of $\phi_{\nu}(x)$ from $\phi_{0}(x)$ in $H^{1}(\mathbb{R})$ for sufficiently small $\nu$, such that $F\left(\phi_{\nu}, \nu\right)=0$ and $\phi_{\nu}(x) \rightarrow \phi_{0}(x)$ in $H^{1}(\mathbb{R})$ as $\nu \rightarrow 0$.

In other words, we have proved above that if a gap soliton exists for $\Gamma_{+}=\Gamma_{-}$and $\omega \in \mathbb{R} \backslash \Sigma$ and the linearized operator $\mathcal{L}$ is non-degenerate, then the gap soliton is uniquely continued into the SGS for small non-zero 
$\left|\Gamma_{+}-\Gamma_{-}\right|$. We confirm this prediction via numerical analysis of the ODE (2.3) with $V(x)$ in (2.8) (i) for $\omega$ taken in the semi-infinite band gap and the first two finite gaps. Numerical approximations of $\phi_{0}(x)$ for $\Gamma_{+}=\Gamma_{-}$are obtained from the Newton-Raphson iterations and the homotopy continuation method. The initial guess for the Newton's iteration is taken from an asymptotic expansion leading to the NLS approximation 13 when $\omega$ is close to the local bifurcation threshold $\omega_{n}$. After a successful convergence for one such $\omega$ we use a standard homotopy continuation and generate a family of gap solitons $\phi_{0}(x)$ parameterized by $\omega$. The discretization of the ODE (2.3) is based on a fourth order central difference approximation of $\partial_{x x}$ on a truncated domain with zero Dirichlet boundary conditions.

\subsection{Numerical Computations of Surface Gap Solitons}

We now proceed to construct SGS's, i.e. solutions $\phi(x)$ of the second-order ODE (2.3) with $\Gamma_{+} \neq \Gamma_{-}$. When $\phi_{0}(x)$ is obtained for a given value of $\omega$, we can apply the numerical homotopy continuation of the solution by deviating $\Gamma_{-}$from $\Gamma_{+}$. At each step, the SGS $\phi(x)$ is thus found via Newton's iterations. The final value of $\Gamma_{-}$, up to which the iteration converges, is denoted by $\Gamma_{*}$.

Fig. 1 shows the values of $\Gamma_{*}$ for $\Gamma_{+}=+1$ (a) and $\Gamma_{+}=-1$ (b). The computational tolerance in $\Gamma_{*}$ is 0.006 inside the band gaps and 0.002 near the band edges. In the case $\Gamma_{+}=1$, local bifurcations of small-amplitude gap solitons occur from the lower band edges. Fig. 1(a) shows that the SGS's exist in the semi-infinite gap, as well as in the first two frequency gaps. In the case $\Gamma_{+}=-1$, local bifurcations of gap solitons occur from the upper band edges. Fig. 1(b) shows that the SGS's exist in the first and second frequency gaps. The two insets of Fig. 1(a) show that $\Gamma_{*}$ decreases fast as $\omega$ moves away from the edge of the first band and that the convergence $\Gamma_{*} \uparrow 1$ as $\omega \uparrow \omega_{0}$ is smooth. We further see from Fig. 1 that the interval of existence shrinks as $\omega$ approaches the value $\omega_{n}$ for any band edge, where gap solitons undertake a local bifurcation. In addition, the interval of existence is extremely large in the semi-infinite gap $\left(-\infty, \omega_{0}\right)$, but it becomes narrow in the finite gaps $\left(\omega_{2 m-1}, \omega_{2 m}\right)$ for $m \geq 1$.

For comparison, the family of SGS's in the gap $\left(\omega_{1}, \omega_{2}\right)$ exists for $-0.24<$ $\Gamma_{*}<1$ in the case $\Gamma_{+}=+1$ and $-1<\Gamma_{*}<0.47$ in the case $\Gamma_{+}=-1$. The family of SGS's in the gap $\left(\omega_{3}, \omega_{4}\right)$ exists in a very narrow region of $0.92<\Gamma_{*}<1$ in the case $\Gamma_{+}=+1$ and in a bigger interval $-1<\Gamma_{*}<0.37$ in the case $\Gamma_{+}=-1$ (similarly to that in the first gap).

Fig. 2 shows profiles of SGS's which correspond to the twelve points 


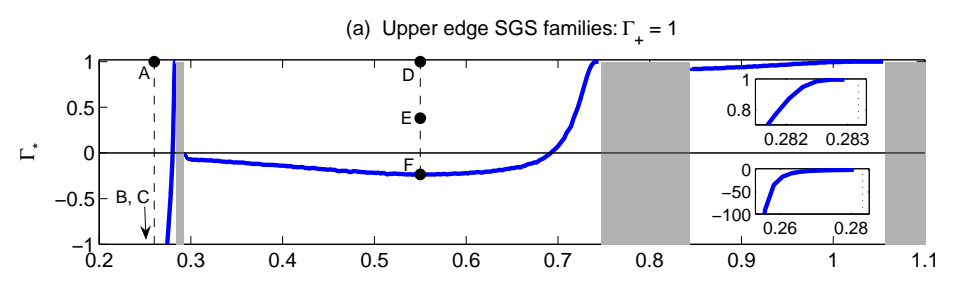

(b) Lower edge SGS families: $\Gamma_{+}=-1$

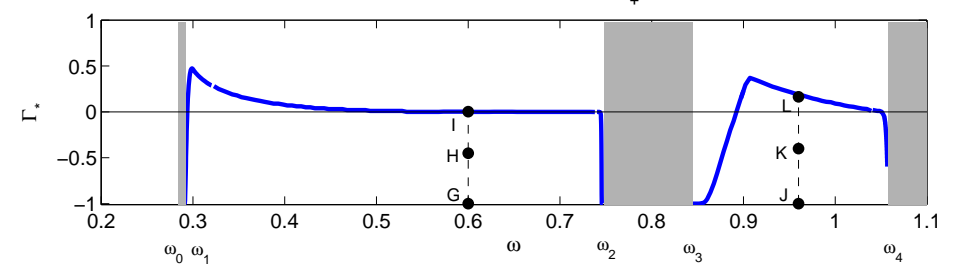

Figure 1: The values of $\Gamma_{*}$ for SGS's originating from symmetric GS families of the first three frequency gaps of $V(x)=\sin ^{2}(\pi x / 10)$. In (a) the upper inset zooms in and the lower inset zooms out on the graph in the semiinfinite gap. The points $A-L$ are referenced in Fig. 2.

labeled $A-L$ in Fig. 1. The full lines correspond to the gap solitons from which the homotopy in $\Gamma_{-}$is started (i.e. points $A, D, G$ and $J$ ). Clearly, the total power and maximum amplitude of the SGS increase as $\left|\Gamma_{+}-\Gamma_{-}\right|$ increases. Also notice that the profiles become more concentrated on the half $x>0$ in the case $\Gamma_{+}=+1$ [see Fig. 2 (a-b)] and on the half $x<0$ in the case $\Gamma_{+}=-1$ [see Fig. $\left.2(\mathrm{c}-\mathrm{d})\right]$ as $\left|\Gamma_{+}-\Gamma_{-}\right|$increases. This is in accord with the law of refraction: when $\Gamma_{+}=+1$ and $\Gamma_{-}$decreases from 1 , the half $x>0$ becomes relatively more focusing and therefore attracts more energy of the soliton, while when $\Gamma_{+}=-1$ and $\Gamma_{-}$increases from -1 , the situation is opposite.

\subsection{Asymptotic Analysis near Gap Soliton Bifurcation Points}

We shall explain now why the existence interval shrinks to zero when $\omega$ approaches the value $\omega_{n}$ where a local bifurcation of gap solitons occurs. As $\omega \rightarrow \omega_{n}$, we have $\left\|\phi_{0}\right\|_{L^{\infty}} \rightarrow 0$ and $\mathcal{L} \rightarrow\left(L-\omega_{n}\right)$. Since the operator $\left(L-\omega_{n}\right)$ is not invertible, the Implicit Function Theorem can not be used and the solution $\phi_{0}(x)$ can not be continued beyond $\nu=0$. In order to give a more precise explanation of this phenomenon, we adopt the NLS approximation for local bifurcation of gap solitons from [13] (see also review in [12]). In 

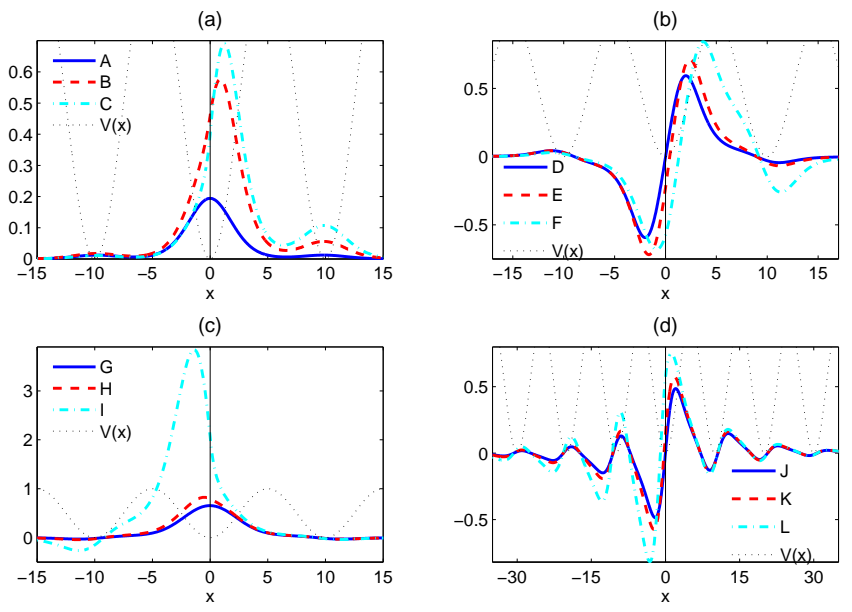

Figure 2: The profiles of SGS's corresponding to the points $A-L$ in Fig. 1 . Values of $\omega$ are $A-C: 0.26, D-F: 0.55, G-I: 0.6, J-L: 0.96$. Values of $\Gamma_{-}$are $A: 1, B:-3.9, C:-15.3, D: 1, E: 0.38, F:-0.235, G:-1, H:-0.45$, $I: 0.002, J:-1, K:-0.4, L: 0.164$.

particular, we consider an asymptotic solution to the ODE (2.3):

$$
\begin{aligned}
& \omega=\omega_{n}+\varepsilon^{2} \Omega+\mathcal{O}\left(\varepsilon^{4}\right), \\
& \phi(x)=\varepsilon A(X) \psi_{n}(x)+\varepsilon^{2} A^{\prime}(X) \tilde{\psi}_{n}(x)+\varepsilon^{3} \phi^{(3)}(x, X)+\mathcal{O}\left(\varepsilon^{4}\right),
\end{aligned}
$$

where $X=\varepsilon x, \varepsilon<<1$, the function $A(X)$ and parameter $\Omega$ are defined below; $\psi_{n}$ and $\tilde{\psi}_{n}$ are the $d$-periodic (or $d$-antiperiodic) Bloch function and generalized Bloch functions respectively of the Hill's equation (2.5) for $\omega=$ $\omega_{n}$, such that

$$
\left(L-\omega_{n}\right) \psi_{n}=0, \quad\left(L-\omega_{n}\right) \tilde{\psi}_{n}=2 \psi_{n}^{\prime} .
$$

The correction term $\phi^{(3)}(x, X)$ at $\mathcal{O}\left(\varepsilon^{3}\right)$ solves the non-homogeneous problem

$$
\left(L-\omega_{n}\right) \phi^{(3)}=\Omega A \psi_{n}+A^{\prime \prime} \psi_{n}+2 A^{\prime \prime} \tilde{\psi}_{n}^{\prime}+\Gamma(X) A^{3} \psi_{n}^{3} .
$$

To ensure boundedness of $\phi^{(3)}(x, X)$ with respect to the variable $x$, and, hence, legitimacy of the expansion (3.3), one has to apply the Fredholm alternative which imposes the orthogonality condition of the right-hand-side of (3.5) with respect to $\psi_{n}(x)$ on $x \in[0, d]$. The orthogonality condition is written as follows

$$
\Omega A+\mu A^{\prime \prime}+\rho \Gamma(X) A^{3}=0,
$$


where

$$
\mu=1+2 \frac{\left(\tilde{\psi}_{n}^{\prime}, \psi_{n}\right)}{\left(\psi_{n}, \psi_{n}\right)}, \quad \rho=\frac{\left(\psi_{n}^{2}, \psi_{n}^{2}\right)}{\left(\psi_{n}, \psi_{n}\right)},
$$

and we have used the standard $L^{2}$ inner product $(\cdot, \cdot)$ over one period $x \in$ $[0, d]$. It is shown in [13] that $\mu=\frac{1}{2} \omega_{2 n, 2 n+1}^{\prime \prime}(k)$ with either $k=0$ or $k=\frac{\pi}{d}$ at the point $\omega=\omega_{n}$, where $\omega_{2 n, 2 n+1}(k)$ is the dispersion relation between $\omega \in\left[\omega_{2 n}, \omega_{2 n+1}\right]$ and $k \in\left[0, \frac{\pi}{d}\right]$.

Due to the nature of the nonlinearity interface, the function $\Gamma(X)$ is the same as $\Gamma(x)$, i.e. $\Gamma(X)=\Gamma_{ \pm}$for $\pm X>0$. We shall prove that no localized solution of the ODE (3.6) exists under the condition $\Gamma_{-} \neq \Gamma_{+}$. Indeed, consider the Hamiltonian of the ODE (3.6):

$$
H[A]=\frac{1}{2}\left[\mu\left(A^{\prime}\right)^{2}+\Omega A^{2}\right]+\frac{1}{4} \rho \Gamma(X) A^{4} .
$$

If $A(X)$ solves the ODE (3.6), then

$$
\frac{d}{d X} H[A(X)]=\frac{1}{4} \rho \Gamma^{\prime}(X) A^{4}(X)=\frac{1}{4} \rho\left(\Gamma_{+}-\Gamma_{-}\right) \delta(X) A^{4}(X),
$$

where $\delta(X)$ is the Dirac delta-function. If $A(X)$ is a localized solution on $X \in \mathbb{R}$, then the integration on $X \in \mathbb{R}$ gives the constraint

$$
0=\lim _{x \rightarrow+\infty} H[A(X)]-\lim _{x \rightarrow-\infty} H[A(X)]=\frac{1}{4} \rho\left(\Gamma_{+}-\Gamma_{-}\right) A^{4}(0),
$$

since $H[A(X)] \rightarrow 0$ if $A(X), A^{\prime}(X) \rightarrow 0$ as $|X| \rightarrow \infty$. Therefore, $A(0)=0$ if $\Gamma_{+} \neq \Gamma_{-}$. Consider now $H[A(X)]$ on $X>0$. It is clear from the decaying conditions as $X \rightarrow \infty$ that $H[A(X)]=$ const $=0$, which together with the fact $A(0)=0$ leads to $0=\lim _{X \downarrow 0} H[A(X)]=\frac{1}{2} \mu\left|A^{\prime}(0)\right|^{2}$, such that $A^{\prime}(0)=0$.

The only solution of the ODE (3.6) with $A(0)=A^{\prime}(0)=0$ is the zero solution $A(X) \equiv 0$.

If $\Gamma_{+}=\Gamma_{-}=\Gamma_{0}$ and $\operatorname{sign}(\mu)=\operatorname{sign}\left(\rho \Gamma_{0}\right)=-\operatorname{sign}(\Omega)$, the ODE (3.6) has the standard sech-soliton decaying as $|X| \rightarrow \infty$. However, the result above shows that the sech-soliton with $\Gamma_{+}=\Gamma_{-}$cannot be homotopically continued to a decaying solution of (3.6) for $\Gamma_{+} \neq \Gamma_{-}$. This proves that $\Gamma_{*} \rightarrow \Gamma_{+}$as $\omega \rightarrow \omega_{n}$ where $\omega_{n}$ is a local bifurcation value.

\section{Bifurcations of surface gap solitons for nons- mooth potentials}

In this section, we study local bifurcations of solutions of the ODE (2.3) when $V(x)$ is a continuous function with the jump in the first derivative 
at the nonlinearity interface. The prototypical example of such potentials is given by (2.8) (ii), where $V_{0}(x)$ is an even potential (in our numerical computations we use $V_{0}$ from (2.6) $)$. We shall consider the existence of SGS's under the normalization $\Gamma_{+}=-\Gamma_{-}=+1$.

\subsection{Surface Gap Soliton Numerical Construction via Gluing}

The point $\left(\delta_{*}, \omega_{*}\right)$ in the parameter domain $\delta \in(0, d)$ and $\omega \in\left(\omega_{2 m-1}, \omega_{2 m}\right)$, $m \in \mathbb{N}$ is defined to be a point of a local bifurcation of SGS's according to the following 2-step algorithm.

\section{(i) Construction of Continuous Solutions}

Let $\phi_{ \pm}(x ; \omega)$ denote the family of single-humped gap solitons parameterized by $\omega \in\left(\omega_{2 m-1}, \omega_{2 m}\right)$ and centered at $x=0$ corresponding to the equation (2.3) with $\Gamma(x) \equiv \Gamma_{ \pm}$respectively. These families bifurcate from the points $\omega=\omega_{2 m}$ for $\Gamma_{+}>0$ and $\omega=\omega_{2 m-1}$ for $\Gamma_{-}<0$. In order to find continuous solutions, we now study for each fixed $\delta \in(0, d)$ the two functions

$$
f_{A}(\omega)=\phi_{-}(-\delta ; \omega)-\phi_{+}(\delta ; \omega), \quad f_{B}(\omega)=\phi_{-}(-\delta ; \omega)+\phi_{+}(\delta ; \omega)
$$

and find their zeros denoted by $\omega_{A, B}=\omega_{A, B}(\delta)$, respectively. For each $\delta$ existence of zeros of either $f_{A}(\omega)$ or $f_{B}(\omega)$ is guaranteed by continuity of $\phi_{ \pm}$as functions of $\omega$ and by the fact that $\phi_{-}\left(-\delta ; \omega_{2 m-1}\right)=\phi_{+}\left(\delta ; \omega_{2 m}\right)=0$ and $\phi_{-}\left(-\delta ; \omega_{2 m}\right) \neq 0, \phi_{+}\left(\delta ; \omega_{2 m-1}\right) \neq 0$. Moreover, several zeros of these functions may occur for the same $\delta$.

When a zero $\omega_{A}(\delta)$ or $\omega_{B}(\delta)$ is found, a $\delta$-parameterized family of continuous solutions $\phi_{A}(x ; \delta)$ or $\phi_{B}(x ; \delta)$, respectively, is constructed by gluing two individual gap solitons

$$
\begin{aligned}
& \phi_{A}(x ; \delta)=\phi_{-}\left(x-\delta ; \omega_{A}\right) \chi_{(-\infty, 0)}+\phi_{+}\left(x+\delta ; \omega_{A}\right) \chi_{[0, \infty)} \\
& \phi_{B}(x ; \delta)=\phi_{-}\left(x-\delta ; \omega_{B}\right) \chi_{(-\infty, 0)}-\phi_{+}\left(x+\delta ; \omega_{B}\right) \chi_{[0, \infty)}
\end{aligned}
$$

The functions $\phi_{A, B}(x ; \delta)$ decay as $|x| \rightarrow \infty$ and are smooth in $x$ everywhere except at the nonlinearity interface $x=0$, where they generally have a jump in the first derivative.

Note that it is important to consider both $\phi_{A}$ and $\phi_{B}$ due to the sign invariance of the ODE (2.3). Each sign produces a branch of continuous solutions of the ODE (2.3).

Figs. 3 (a) and 4 (a) present the numerically computed $\omega_{A, B}(\delta)$ in the gaps $\left(\omega_{1}, \omega_{2}\right)$ and $\left(\omega_{3}, \omega_{4}\right)$ respectively. The lack of smoothness in the curves 
in these figures is due to an insufficient resolution in the search algorithm and can be corrected with a finer resolution. Note that when $\omega_{A, B}(\delta)$ is multiple valued as seen in Fig. 3 (a) and 4 (a), we may have several decaying solutions $\phi_{A}(x)$ and/or $\phi_{B}(x)$ for the same $\delta$.

\section{(ii) Construction of $C^{1}$ Surface Gap Solitons}

Next, we search for continuously differentiable solutions within the above family $\phi_{A, B}(x ; \delta)$. To ensure the continuity of the first derivative of $\phi(x ; \delta)$ at $x=0$, we search for zeros of the two functions

$$
g_{A}(\delta)=\phi_{-}^{\prime}\left(-\delta ; \omega_{A}\right)-\phi_{+}^{\prime}\left(\delta ; \omega_{A}\right), \quad g_{B}(\delta)=\phi_{-}^{\prime}\left(-\delta ; \omega_{B}\right)+\phi_{+}^{\prime}\left(\delta ; \omega_{B}\right) .
$$

If a zero of either $g_{A}(\delta)$ or $g_{B}(\delta)$, denoted by $\delta_{*}$, exists, then the function $\phi_{A}\left(x ; \delta_{*}\right)$ or $\phi_{B}\left(x ; \delta_{*}\right)$, respectively, in (4.1) has a continuous first derivative across the point $x=0$. Figs. 3 (b) and 4 (b) present the numerical results on computing $\delta_{*}$. The labelled intersection points $O, P, Q, R, S$ and $T$ correspond to zeros of $g_{A, B}(\delta)$. They are found as intersection points of solid and dashed curves of the same color. The solid black line shows the plot of $\phi_{+}^{\prime}\left(\delta ; \omega_{A}\right)$ and the dashed black line shows $\phi_{-}^{\prime}\left(-\delta ; \omega_{A}\right)$. Similarly, the solid gray line plots $-\phi_{+}^{\prime}\left(\delta ; \omega_{B}\right)$ and the dashed gray line plots $\phi_{-}^{\prime}\left(-\delta ; \omega_{B}\right)$. Therefore, an intersection of a solid black and a dashed black line (points $O, Q, S)$ gives zeros $\delta_{*}$ of $g_{A}(\delta)$ and, thus, a $C^{1}$ SGS $\phi_{A}\left(x ; \delta_{*}\right)$. Similarly, an intersection of a solid gray and a dashed gray line (points $P, R, T$ ) gives zeros $\delta_{*}$ of $g_{B}(\delta)$ and, thus, a $C^{1} \operatorname{SGS} \phi_{B}\left(x ; \delta_{*}\right)$.

Table 2 shows the approximate computed values of $\delta_{*}$ and corresponding $\omega_{*}=\omega_{A, B}\left(\delta_{*}\right)$ at the points $O-T$ for branches $A, B$ of solutions given by (4.1). Note that additional points $\left(\delta_{*}, \omega_{*}\right)$ can be obtained by generalizing the above functions $f_{A, B}$ and $g_{A, B}$ to

$$
f_{j_{A}}(\omega)=\phi_{-}(-(j d+\delta) ; \omega)-\phi_{+}(j d+\delta ; \omega), \quad f_{j_{B}}(\omega)=\phi_{-}(-(j d+\delta) ; \omega)+\phi_{+}(j d+\delta ; \omega)
$$

and

$g_{j_{A}}(\delta)=\phi_{-}^{\prime}\left(-(j d+\delta) ; \omega_{A}\right)-\phi_{+}^{\prime}\left(j d+\delta ; \omega_{A}\right), \quad g_{j_{B}}(\delta)=\phi_{-}^{\prime}\left(-(j d+\delta) ; \omega_{B}\right)+\phi_{+}^{\prime}\left(j d+\delta ; \omega_{B}\right)$

for $j \in\{1,2, \ldots\}$ with $V$ still defined as in (2.8)(ii). Non-trivial points $\left(\omega_{*}, \delta_{*}\right)$ may exist for any such $j$. For illustration we have found one such point for $j=1$. The computed value is $\left(\omega_{*}, \delta_{*}\right) \approx(0.73,7.33)$ and the resulting SGS corresponds to the point $Z$ in Fig. 5(a). Such additional solutions are SGSs of smaller amplitude compared to those for $j=0$. 
(a)

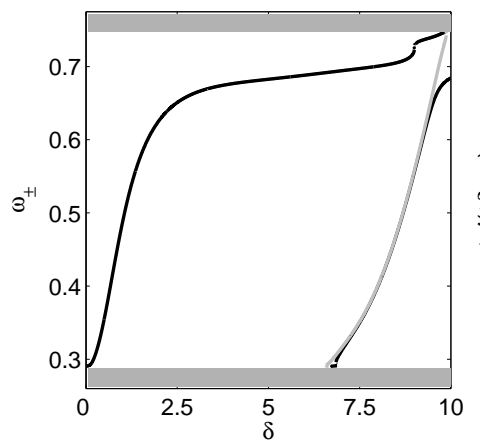

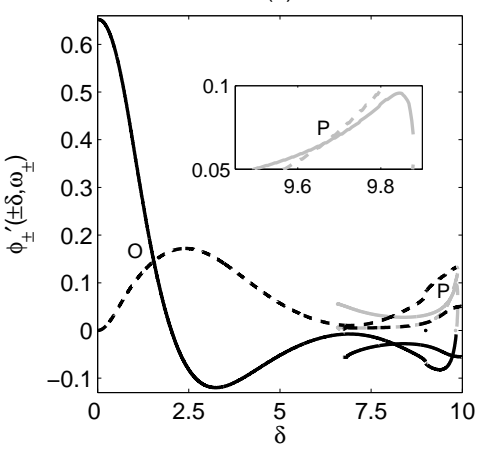

Figure 3: Two-step search for $\left(\omega_{*}, \delta_{*}\right)$ in the gap $\left(\omega_{1}, \omega_{2}\right)$. (a) Result of step (i) - parametrization of the families of continuous solutions (4.1): black line $\omega_{A}(\delta)$, gray line $\omega_{B}(\delta)$; (b) step (ii) - search for $\delta_{*}$ : solid black $\phi_{+}^{\prime}\left(\delta ; \omega_{A}\right)$, dashed black $\phi_{-}^{\prime}\left(-\delta ; \omega_{A}\right)$, solid gray $-\phi_{+}^{\prime}\left(\delta ; \omega_{B}\right)$ and dashed gray $\phi_{-}^{\prime}\left(-\delta ; \omega_{B}\right)$. Labeled points correspond to $C^{1}$ SGS's.

(a)

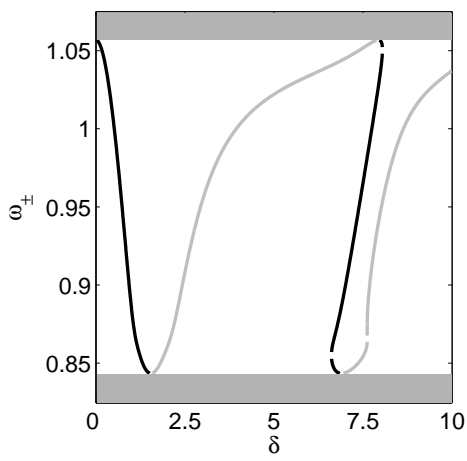

(b)

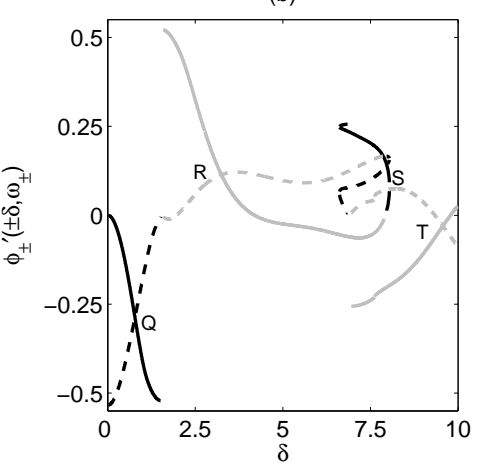

Figure 4: Analogous to Fig. 3 but for the gap $\left(\omega_{3}, \omega_{4}\right)$. 


\begin{tabular}{||c||c|c|c|c|c|c||}
\hline point & $O$ & $P$ & $Q$ & $R$ & $S$ & $T$ \\
\hline branch of solution & A & B & A & B & A & B \\
\hline$\omega_{*}$ & 0.58 & 0.70 & 0.94 & 0.97 & 1.03 & 1.03 \\
\hline$\delta_{*}$ & 1.54 & 9.66 & 0.78 & 3.24 & 7.97 & 9.57 \\
\hline
\end{tabular}

Table 2: Bifurcation points for surface gap solitons in the domain $\omega \in$ $\left(\omega_{1}, \omega_{2}\right) \cup\left(\omega_{3}, \omega_{4}\right)$ and $\delta \in(0, d)$ for branches of solutions given by (4.1).

\subsection{Numerical Homotopy Continuation of SGSs}

Assuming the existence of a point $\left(\omega_{*}, \delta_{*}\right)$, we have constructed the SGS of the ODE (2.3), where the potential function $V(x)$ is given by (2.8) (ii) and $(\omega, \delta)=\left(\omega_{*}, \delta_{*}\right)$. The surface gap soliton denoted as $\phi_{*}(x)$ is represented by one of the functions in (4.1) with $(\omega, \delta)=\left(\omega_{*}, \delta_{*}\right)$. Each of these solutions can be used as a starting point for a numerical homotopy continuation to generate a family of SGS's parameterized by $\omega \subset\left(\omega_{2 m-1}, \omega_{2 m}\right)$ for a given value of $\delta=\delta_{*}$. Similarly, for a fixed $\omega=\omega_{*}$ a family parameterized by $\delta \subset$ $(0, d)$ can be constructed. Under the same assumption that the operator $\mathcal{L}=$ $-\partial_{x}^{2}-\omega_{*}+V(x)-3 \Gamma(x) \phi_{*}^{2}(x)$ is invertible, the Implicit Function Theorem implies that there exists a unique smooth continuation of the particular solution $\phi_{*}(x)$ to the family of solutions along parameters $\omega$ and $\delta$.

We restrict our numerical studies to the continuation in $\omega$. Numerical results of such continuation from the SGS's at points $O-T$ are shown in Figs. [5 (a) and 6 (a). The curves plot the total soliton power $\|\phi\|_{L^{2}(\mathbb{R})}^{2}$ as a function of frequency $\omega$ for fixed $\delta=\delta_{*}$. Note that each curve corresponds to a different value of $\delta_{*}$ and hence a different potential $V(x)$. The values of $\delta_{*}$ can be read in Table 2, Termination of a continuation curve is defined when the total power of the soliton becomes zero or when Newton iteration convergence fails. As the figures show, the latter case is always accompanied by the slope of the continuation curve becoming infinite suggesting a violation of the implicit function theorem assumptions. The former termination case is studied in the following subsection.

\subsection{Analysis of Termination Points of Surface Gap Solitons}

We shall now consider the termination points of the solution families plotted in Figs. 5 (a) and 6 (a) where the soliton power becomes zero. The points are labeled $O_{L}-T_{L}$ and their corresponding $\delta$ and $\omega$ are given in Table 3 ,

The termination points are expected to be related to existence of non- 

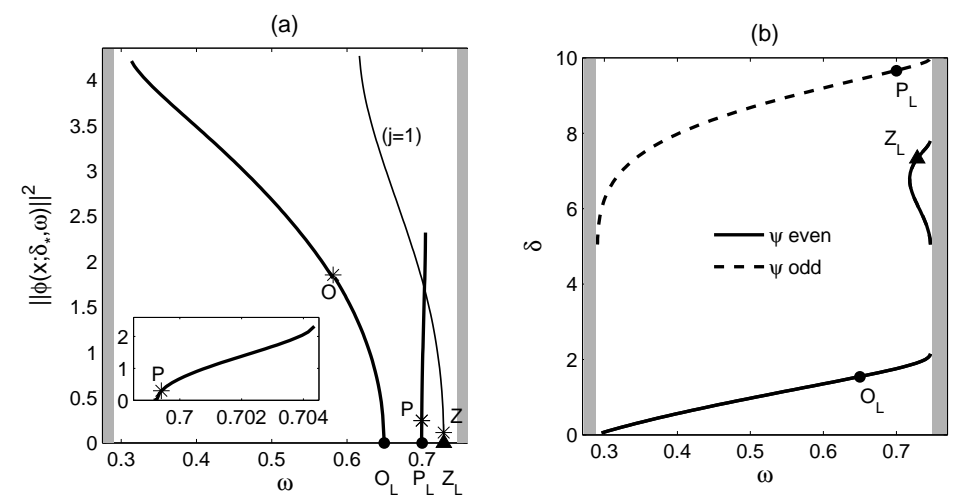

Figure 5: (a) SGS continuation curves, total power versus frequency, in the gap $\left(\omega_{1}, \omega_{2}\right)$. Labeled points $O, P$ correspond to those in Fig. 3 (b). Point $Z$ is discussed in Sec. 4.1. Points $O_{L}, P_{L}$ and $Z_{L}$ are SGS termination points. (b) Point spectrum of the linear Schrödinger operator inside $\left(\omega_{1}, \omega_{2}\right)$ for all $\delta \in(0, d)$. Full/dashed lines: eigenvalues with even/odd eigenfunctions.
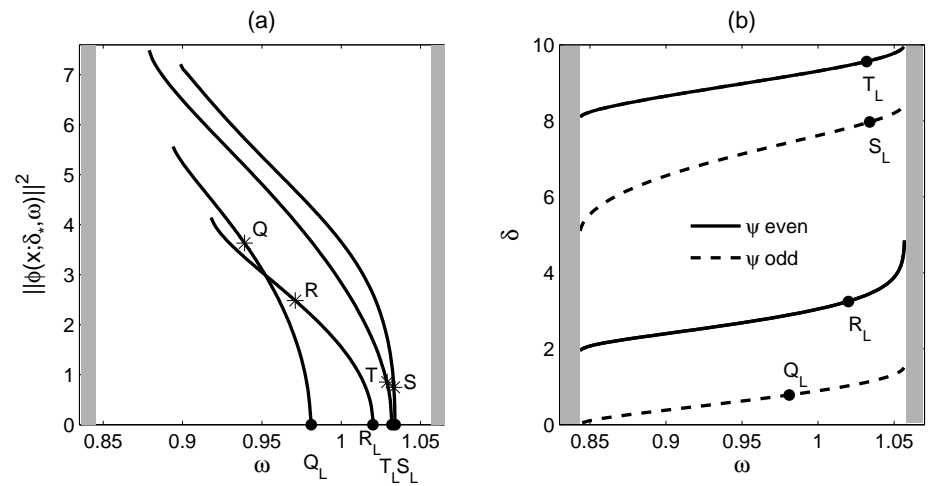

Figure 6: (a) SGS continuation curves, total power versus frequency, in the gap $\left(\omega_{3}, \omega_{4}\right)$. Labeled points $Q-T$ correspond to those in Fig. 4 (b). Points $Q_{L}-T_{L}$ are SGS termination points. (b) Point spectrum of the linear Schrödinger operator inside $\left(\omega_{3}, \omega_{4}\right)$ for all $\delta \in(0, d)$. Full/dashed lines: eigenvalues with even/odd eigenfunctions. 


\begin{tabular}{||c||c|c|c|c|c|c||}
\hline point & $O_{L}$ & $P_{L}$ & $Q_{L}$ & $R_{L}$ & $S_{L}$ & $T_{L}$ \\
\hline$\delta$ & 1.54 & 9.66 & 0.78 & 3.24 & 7.97 & 9.57 \\
\hline$\omega$ & 0.65 & 0.70 & 0.98 & 1.02 & 1.03 & 1.03 \\
\hline
\end{tabular}

Table 3: Termination points for the six SGS families in Figs. 5 (a) and 6 (a).

trivial bound states in the (point) spectrum of the Schrödinger operator for the same potential $V(x)$, i.e. with exponentially decaying solutions of the linear ODE

$$
-\psi^{\prime \prime}-\omega \psi+V(x) \psi=0, \quad \psi: \mathbb{R} \mapsto \mathbb{R},
$$

for $V(x)$ in (2.8) (ii) and $\omega \in \mathbb{R} \backslash \Sigma$. The point spectrum is nonempty due to the singularity of $V(x)$ at $x=0$.

\subsubsection{Numerical Results}

Results of numerical computations of the point spectrum contained in the first two finite gaps $\left(\omega_{1}, \omega_{2}\right)$ and $\left(\omega_{3}, \omega_{4}\right)$ are shown in Figs. 5 (b) and 6 (b) for all values $\delta \in[0, d]$. The eigenfunctions $\psi$ are either even (full lines) or odd (dashed lines). For the six values of $\delta$ corresponding to the SGS families in Figs. 5 (a) and 6 (a) the eigenvalues are marked by black dots and are in perfect agreement with the values of $\omega$ at the termination points $O_{L}-T_{L}$. The symmetry (even/odd) of the bound states at $O_{L}-T_{L}$ also matches that of the eigenfunctions at the marked points in the point spectrum. The eigenvalue curve originating as well as ending at $\omega_{2}$ in Fig. 5(b) corresponds to the termination point $Z_{L}$ of the SGS family for $j=1$ in Fig. 5 (a). The termination point $Z_{L}$ for the same value of $\delta$ is shown by a triangle.

\subsubsection{Bifurcation Analysis for $|\delta|$ Small}

In the remaining part we consider bifurcations of point spectrum of the Schrödinger operator from the band edges for small values of $|\delta|$ (or, due to the $d$ periodicity of $V$, equivalently for $\delta$ near 0 from above and near $d$ from below). This analysis will prove the existence of the spectral curves near $\delta=0$ and $\delta=10$ in Figs. 5] (b) and 6 (b), i.e. the existence of curves with points $O_{L}$ and $Q_{L}$ locally to $\delta=0$ and the curves with points $P_{L}$ and $T_{L}$ locally to $\delta=10$. 
In order to construct solutions of the spectral problem (4.2), we first consider exponentially decaying solutions of the ODE on the half-line

$$
-\psi_{+}^{\prime \prime}-\omega \psi_{+}+V_{0}(x+\delta) \psi_{+}=0, \quad \psi_{+}: \mathbb{R}_{+} \mapsto \mathbb{R} .
$$

By using the fundamental solution of the Hill's equation (2.5), we can express $\psi_{+}(x)$ in the form $\psi_{+}=e^{-\kappa x} u_{-}(x+\delta)$, where $u_{-}(x)$ are periodic or antiperiodic bounded solutions of the Hill's equation (2.5) with $V(x)=V_{0}(x)$, i.e. solutions of (2.5) at a band edge $\omega=\omega_{n}$.

As $V(x)$ is even, the function $\psi_{+}(x)$ admits a symmetric (even) reflection about $x=0$ if $\psi_{+}^{\prime}(0)=0$, which is equivalent to the condition

$$
G_{1}(\delta, \kappa)=u_{-}^{\prime}(\delta)-\kappa u_{-}(\delta)=0,
$$

and it admits an anti-symmetric (odd) reflection about $x=0$ if $\psi_{+}(0)=0$, which is equivalent to the condition

$$
G_{2}(\delta, \kappa)=u_{-}(\delta)=0 .
$$

Since eigenvalues of the spectral problem (4.2) are simple and the eigenfunctions are either even or odd, all eigenvalues of the spectral problem (4.2) in the band gaps $\omega \in \mathbb{R} \backslash \Sigma$ are defined by zeros of the functions $G_{1}(\delta, \kappa)$ and $G_{2}(\delta, \kappa)$ in $\kappa$ for a given value of $\delta$, where $\kappa \geq 0$ and the values of $\kappa$ are related to the values of $\omega$ in the band gaps. Both functions $G_{1,2}$ are analytic in $\delta \in \mathbb{R}$ and periodic with period $d$. Both functions admit analytic continuation in the parameter $\kappa \in \mathbb{R}_{+}[8$.

If $\delta=0$, the only zeros of $G_{1}(\delta, \kappa)$ and $G_{2}(\delta, \kappa)$ occur at $\kappa=0$, i.e. at the band edges $\omega=\omega_{n}$. Indeed, if $G_{1}(0, \kappa)=0$, then $\psi_{+}^{\prime}(0)=0$, such that $\psi_{+}(x)=\psi_{n}(x)$ is an even function on $x \in \mathbb{R}$. However, $\psi_{+}(x)$ decays exponentially as $x \rightarrow \infty$ and grows exponentially as $x \rightarrow-\infty$ if $\kappa>0$. Therefore, $G_{1}(0, \kappa)=0$ is equivalent to $\kappa=0$. A similar argument works for $G_{2}(0, \kappa)=0$.

(i) Bifurcation of Even Eigenfunctions

Let us first consider the zeros of $G_{1}(\delta, \kappa)$. Computing the derivatives of $G_{1}(\delta, \kappa)$ in $\delta$ and $\kappa$ at $(\delta, \kappa)=(0,0)$, we obtain

$$
\begin{aligned}
& \partial_{\delta} G_{1}(0,0)=u_{-}^{\prime \prime}(0)=\psi_{n}^{\prime \prime}(0)=\left(V_{0}(0)-\omega_{n}\right) \psi_{n}(0), \\
& \partial_{\kappa} G_{1}(0,0)=-\tilde{\psi}_{n}^{\prime}(0)-\psi_{n}(0),
\end{aligned}
$$

where $\tilde{\psi}_{n}$ is the generalized Bloch function, see (3.4). The fact $\tilde{\psi}_{n}=$ $-\left.\frac{\partial u_{-}}{\partial \kappa}\right|_{\kappa=0}$ is clear from differentiation of (2.5) with respect to $\kappa$. 
It is found in [12] that

$$
D(x)=\psi_{n}(x) \tilde{\psi}_{n}^{\prime}(x)-\psi_{n}^{\prime}(x) \tilde{\psi}_{n}(x)+\psi_{n}^{2}(x)
$$

is constant in $x$, i.e. $D(x)=D(0)$, and that

$$
D(0)=\frac{1}{2} \omega_{2 n-1,2 n}^{\prime \prime}(k)\left(\psi_{n}, \psi_{n}\right),
$$

where either $k=0$ or $k=\frac{\pi}{d}$ at the bifurcation point $\omega=\omega_{n}$. Since $\psi_{n}^{\prime}(0)=$ $0, D(0)=\psi_{n}(0)\left(\tilde{\psi}_{n}^{\prime}(0)+\psi_{n}(0)\right)$ and the leading-order approximation for the root of $G_{1}(\delta, \kappa)$ near $(\delta, \kappa)=(0,0)$ is given by

$$
\delta=\frac{\tilde{\psi}_{n}^{\prime}(0)+\psi_{n}(0)}{\psi_{n}(0)\left(V_{0}(0)-\omega_{n}\right)} \kappa+\mathcal{O}\left(\kappa^{2}\right)=\frac{D(0)}{\psi_{n}^{2}(0)\left(V_{0}(0)-\omega_{n}\right)} \kappa+\mathcal{O}\left(\kappa^{2}\right),
$$

where $\psi_{n}(0) \neq 0\left(\right.$ since $\left.\psi_{n}^{\prime}(0)=0\right)$. Using (4.3) and the facts $\omega_{n}>0$ and $V_{0}(0)=0$ for the numerical example (2.6), we get

$$
\delta=-\frac{\omega_{2 n-1,2 n}^{\prime \prime}(k)\left(\psi_{n}, \psi_{n}\right)}{2 \psi_{n}^{2}(0) \omega_{n}} \kappa+\mathcal{O}\left(\kappa^{2}\right)
$$

Therefore, the bifurcation occurs for $\delta>0$ if $\omega_{2 n-1,2 n}^{\prime \prime}(k)<0$ (e.g. for $\omega$ to the right of $\left.\omega_{1}\right)$ and for $\delta<0$ if $\omega_{2 n-1,2 n}^{\prime \prime}(k)>0$ (e.g. for $\omega$ to the left of $\omega_{0}$ and $\left.\omega_{4}\right)$, see Table 1 . Note that the negative values of $\delta$ correspond to the values of $\delta$ below the level $\delta=d$ due to periodicity of the function $G_{1}(\delta, \kappa)$ in $\delta$. The above local existence analysis for even bound states is confirmed by the full lines near $\delta=0$ in Fig. [5 (b) and near $\delta=d=10$ in Fig. 6 (b).

(ii) Bifurcation of Odd Eigenfunctions

Similarly to (i), we study the zeros of $G_{2}(\delta, \kappa)$. We compute the derivatives of $G_{2}(\delta, \kappa)$ in $\delta$ and $\kappa$ at $(\delta, \kappa)=(0,0)$

$$
\begin{aligned}
& \partial_{\delta} G_{2}(0,0)=u_{-}^{\prime}(0)=\psi_{n}^{\prime}(0), \\
& \partial_{\kappa} G_{2}(0,0)=-\tilde{\psi}_{n}(0),
\end{aligned}
$$

such that the leading-order approximation for the root of $G_{2}(\delta, \kappa)$ near $(\delta, \kappa)=(0,0)$ is given by

$$
\delta=\frac{\tilde{\psi}_{n}(0)}{\psi_{n}^{\prime}(0)} \kappa+\mathcal{O}\left(\kappa^{2}\right)=-\frac{\omega_{2 n-1,2 n}^{\prime \prime}(k)\left(\psi_{n}, \psi_{n}\right)}{2\left(\psi_{n}^{\prime}(0)\right)^{2}} \kappa+\mathcal{O}\left(\kappa^{2}\right),
$$

where $\psi_{n}^{\prime}(0) \neq 0$ and $D(0)=-\psi_{n}^{\prime}(0) \tilde{\psi}_{n}(0)$ (since $\left.\psi_{n}(0)=0\right)$. Therefore, the bifurcation occurs for $\delta>0$ if $\omega_{2 n-1,2 n}^{\prime \prime}(k)<0$ (e.g. for $\omega$ to the right 
of $\left.\omega_{3}\right)$ and for $\delta<0$ if $\omega_{2 n-1,2 n}^{\prime \prime}(k)>0$ (e.g. for $\omega$ to the left of $\omega_{2}$ ). The dashed lines near $\delta=0$ in Fig. 6 (b) and near $\delta=d=10$ in Fig. 5] (b) confirm this analysis.

Note that there are curves in Figs. [5(b) and 6 (b) which do not bifurcate from $\delta=0$ and $\delta=d=10$ but still bifurcate from the band edge $\omega=$ $\omega_{n}$. Bifurcations of these curves cannot be confirmed from the analytical theory above, unless the values of $G_{1,2}(\delta ; 0)$ for $0<\delta<d$ are approximated numerically.

\section{Conclusion}

We have employed methods of bifurcation theory for the existence problem of SGS's supported by the nonlinearity interface and the periodic potential. Two bifurcation problems are considered numerically. The first bifurcation takes place from the standard gap solitons existing at the zero jump of the nonlinearity coefficient. The second bifurcation takes place from the bound state consisting of parts of two standard gap solitons glued together in a continuously differentiable SGS. Three asymptotic results are described in the article. We show that the standard gap solitons can be continued generally for small jumps in the nonlinearity coefficient. On the contrary, no SGS's for non-zero jump of the nonlinearity coefficient exists in the NLS approximation which is valid near the band edges. In addition, we study analytically bifurcations of eigenvalues of the Schrödinger operator with nonsmooth potential from band edges of the Hill's equation.

One can argue that the SGS's bifurcating from a standard gap soliton or a gluing combination of two gap solitons inherits stability properties of gap solitons in the neighborhood of the local bifurcation points. Stability of standard gap solitons was considered analytically and numerically in [13. The stability properties can change far from the bifurcation points. Detailed computatitons of stability of the SGS's will be the subject of the forthcoming work.

Acknowledgement. T.D. is supported by ETH Research Fellowship. D.P. is supported by the Humboldt Research Fellowship hosted at Institut

für Analysis, Dynamik und Modellierung, Fakultaet für Mathematik und Physik at the Universität Stuttgart. He thanks people at ETH Zurich for hospitality during his visit. 


\section{References}

[1] D. Blömer, A. Szameit, F. Dreisow, T. Schreiber, S. Nolte, and A. Tünnermann, "Nonlinear refractive index of fs-laser-written waveguides in fused silica," Opt. Express 14, 2151-2157 (2006)

[2] M.S. Eastham, The Spectral Theory of Periodic Differential Equations, (Scottish Academic Press, Edinburgh, 1973)

[3] G. Fibich, Y. Sivan, and M. I. Weinstein, "Bound states of nonlinear Schrödinger equations with a periodic nonlinear microstructure," Physica D 217, 31-57 (2006)

[4] J. Hudock, S. Suntsov, D. Christodoulides, and G. Stegeman, "Vector discrete nonlinear surface waves," Opt. Express 13, 7720-7725 (2005)

[5] Y.V. Kartashov, A.A. Egorov, V.A. Vysloukh, and L. Torner, "Surface vortex solitons," Opt. Express 14, 4049-4057 (2006)

[6] Y.V. Kartashov and L. Torner, "Multipole-mode surface solitons," Opt. Lett. 31, 2172-2174 (2006).

[7] Y.V. Kartashov, V.A. Vysloukh and L. Torner, "Surface gap solitons", Phys. Rev. Lett. 96, 073901 (2006)

[8] W. Kohn, "Analytic properties of Bloch waves and Wannier functions", Phys. Rev. 115, 809-821 (1959)

[9] W. Magnus and S. Winkler, Hill's equation, Interscience Tracts in Pure and Applied Mathematics, No. 20 (John Wiley \& Sons, New YorkLondon-Sydney, 1966)

[10] K.G. Makris, S. Suntsov, D.N. Christodoulides, G.I. Stegeman, and A. Hache, "Discrete surface solitons," Opt. Lett. 30, 2466-2468 (2005)

[11] A. Pankov, "Periodic nonlinear Schrdinger equation with application to photonic crystals", Milan J. Math. 73, 259-287 (2005)

[12] D. Pelinovsky, "Asymptotic reductions of the Gross-Pitaevskii equation", in Emergent nonlinear phenomena in Bose-Einstein condensates: Theory and Experiment, Eds. P. Kevrekidis, D. Frantzeskakis, and R. Carretero (Springer, Heidelberg, 2007) 
[13] D.E. Pelinovsky, A.A. Sukhorukov, and Y. Kivshar, "Bifurcations and stability of gap solitons in periodic structures," Phys. Rev. E 70, 036618 (2004)

[14] B. Simon and M. Reed, Methods of Modern Mathematical Physics IV: Analysis of Operators, (Academic Press, New York, 1978)

[15] Y. Sivan, G. Fibich, and M. I. Weinstein, "Waves in nonlinear lattices - ultrashort optical pulses and Bose-Einstein condensates," Phys. Rev. Lett. 97, 193902 (2006)

[16] S. Suntsov, K. G. Makris, D. N. Christodoulides, G. I. Stegeman, A. Hache, R. Morandotti, H. Yang, G. Salamo, and M. Sorel, "Observation of Discrete Surface Solitons," Phys. Rev. Lett. 96, 063901 (2006)

[17] W. J. Tomlinson, "Surface wave at a nonlinear interface," Opt. Lett. 5, 323-325 (1980) 